\title{
ARTICLE
}

\section{Zigzag line defects and manipulation of colloids in a nematic liquid crystal in microwrinkle grooves}

Takuya Ohzono $^{1}$ \& Jun-ichi Fukuda ${ }^{1}$

Spatially confined liquid crystals exhibit non-uniform alignment, often accompanied by selforganised topological defects of non-trivial shape in response to imposed boundary conditions and geometry. Here we show that a nematic liquid crystal, when confined in a sinusoidal microwrinkle groove, exhibits a new periodic arrangement of twist deformations and a zigzag line defect. This periodic ordering results from the inherent liquid crystal elastic anisotropy and the antagonistic boundary conditions at the flat liquid crystal-air and the curved liquid crystalgroove interfaces. The periodic structure can be tuned by controlling the groove geometry and the molecular chirality, which demonstrates the importance of boundary conditions and introduced asymmetry for the engineering of topological defects. Moreover, the kinks in the zigzag defects can trap small particles, which may afford a new method for manipulation of colloids. Our system, which uses easily fabricated microwrinkle grooves, provides a new microfabrication method based on the arrangement of controllable defects.

\footnotetext{
${ }^{1}$ Nanosystem Research Institute (NRI), National Institute of Advanced Industrial Science and Technology (AIST), 1-1-1 Higashi, Tsukuba 305-8565, Japan. Correspondence and requests for materials should be addressed to T.O. (email: ohzono-takuya@aist.go.jp).
} 
$\mathrm{N}$ ematic (N) liquid crystals (LCs) are anisotropic fluids with long-range molecular orientational order and no positional order of their constituent molecules ${ }^{1}$. The local orientational order of an NLC is specified by a unit vector, $\boldsymbol{n}$, known as the director. The free energy of an NLC in terms of $n$, called the Frank elastic energy, is expressed by $F=(1 / 2) \int \mathrm{d} \mathbf{r}\left[K_{11}(\operatorname{div} \boldsymbol{n}(\mathbf{r}))^{2}+\right.$ $\left.K_{22}(\boldsymbol{n}(\mathbf{r}) \cdot \operatorname{curl} \boldsymbol{n}(\mathbf{r}))^{2}+K_{33}(\boldsymbol{n}(\mathbf{r}) \times \operatorname{curl} \boldsymbol{n}(\mathbf{r}))^{2}\right]$, where $K_{11}, K_{22}$ and $K_{33}$ are elastic constants for splay, twist and bend distortions, respectively. The static profile of the director is determined by minimising the Frank elastic energy under the boundary conditions imposed at the surfaces confining the NLC. The geometry of the confining surfaces and the interaction between a surface and NLC molecules, called the surface anchoring, have a crucial role in the control of LC alignment. Although a uniform $\boldsymbol{n}$ would minimize the Frank elastic energy density in the bulk, various distorted alignments appear when the LC is confined in specific geometries, such as the twisted $\mathrm{N}$ cell, hybrid alignments ${ }^{2-7}$, capillary tubes ${ }^{8-10}$, emulsion droplets $^{11,12}$, thin cells ${ }^{13}$ and shells ${ }^{14}$. Patterned surfaces ${ }^{15-17}$ are also used to induce regularly distorted alignments. The resulting distortions, which are often periodic or include topological defects, make LCs useful for electro-optical applications, assembling colloids ${ }^{18,19}$ and for the study of topological problems in a vector field ${ }^{20,21}$.

In a typical hybrid-alignment system, where one of the two confining surfaces imposes normal anchoring, and the other imposes planar anchoring, a topological defect line, known as a disclination, generally forms during the equilibration and is stabilized by a nonflat boundary ${ }^{7}$. When the director $\boldsymbol{n}$ is perpendicular to a nearby disclination line, the disclination is called a wedge disclination, and the winding number is well defined; in this case, it is $\pm 1 / 2$. A halfinteger winding number is allowed by the equivalence of $\boldsymbol{n}$ and $-\boldsymbol{n}$ in an NLC.

In this paper, we consider an NLC in a microgroove, where the NLC aligns normal to the flat air-LC interface, and the curved LCgroove interface imposes a planar alignment with its easy axis perpendicular to the groove direction. We utilize a recently developed method to manipulate liquids into microfilaments in the curved grooves of self-organized microwrinkles ${ }^{22}$. We have discovered a novel symmetry-broken, self-organized structure with periodic domains of alternating twist directions and a zigzag disclination line with a winding number of $+1 / 2$. The geometrical arrangement in a previous study reporting a zigzag disclination ${ }^{9,10}$ is entirely different from ours; a flat capillary tube employed there does not allow the NLC to come into contact with air, and the surface of the tube imposes normal alignment. Moreover, our microwrinkle system allows the geometrical parameters, such as the groove wavelength in the micrometre range, to be easily controlled. We propose that the non-trivial configuration arises from the intrinsic anisotropy in the elasticity of an NLC with $K_{11} \neq K_{22} \neq K_{33}$, and that a smaller $K_{22}$ yields a spontaneous twist deformation below the disclination, which rotates it away from the groove direction.

\section{Results}

Observation of twisted domains with zigzag line defects. Our microwrinkle surface ${ }^{22-25}$ (see Methods) has a sinusoidal undulating surface with a specific wavelength $\lambda$, and a depth $D$; the grooves can serve as open capillaries for a liquid ${ }^{22}$. The liquid fills about threequarters of $\lambda$ (Fig. 1a, Supplementary Fig. S1) and is called a liquid filament. A typical NLC, pentylcyanobiphenyl (5CB), was confined as liquid filaments. The air-NLC interface is almost flat in contrast to the bottom-curved surface of the wrinkle groove. The maximum thickness of the liquid filament at the centre, $h$, is slightly smaller than $D$.

The air-NLC interface imposes normal alignment, where the tilt angle measured from the $z$ axis $\theta \approx 0$, and the azimuthal angle measured from the $x$ axis, $\phi$, can take any value (Fig. 1a). In contrast, the wrinkle surface imposes planar alignment, where $\theta \approx \pi / 2$ and $\phi \approx 0$, or $\pi$, with the easy axis direction perpendicular to the groove direction (Supplementary Fig. S2). The local easy axis is in the $x-z$ plane and along the tangential direction of the sinusoidal curve.

Symmetry-broken, periodic optical rotations were observed along the groove direction with a periodicity $\Lambda$ (Fig. lb-e). Fig. If shows the proposed alignment structure from the analysis of the optical images (Supplementary Fig. S3); $\theta$ decreases from $\pi / 2$ to 0 from the bottom to the top of the groove; therefore, the optical rotation, which reaches a maximum in the centre of the domain, can be attributed to twist distortions in the bottom half, $\phi \approx \pm 25^{\circ}$. The black zigzag line that deviates from the $y$ axis in Fig. 1b,d is a disclination line that changes its direction at the domain boundaries, separating domains with opposite twist handedness. A different observation of an inclined sample shown in Supplementary Fig. S4 clearly demonstrates that the winding number of the disclination line is positive, $+1 / 2$. Obviously, with our concaved wrinkle groove with planar anchoring, $a+1 / 2$ disclination line involves smaller splaybend distortions than a $-1 / 2$ one. The angle of the disclination line from the $y$ axis, $\Omega \approx \pm 8^{\circ}$ and $\phi \Omega>0$, suggesting that the local direction of the disclination line and the twisted local director tend to make a smaller angle rather than retain a right angle (the sign of $\Omega$ is defined so that a clockwise rotation gives a positive value of $\Omega$; see Fig. 1f). This periodic domain structure is stable at room temperature $(\sim 300 \pm 5 \mathrm{~K})$ for at least a month, unless the LC evaporates.

The periodic domain structure is not observed when $\lambda$ is below $\sim 1.5 \mu \mathrm{m}$ (Fig. $1 \mathrm{~g}$ ), or $h<150 \mathrm{~nm}$. If $h$ is much smaller than the characteristic length associated with surface anchoring, $R_{\mathrm{c}} \approx K / W$ $\approx 1 \mu \mathrm{m}$ (refs 11,21$) \quad\left(K \approx 10^{-11} \mathrm{~N}\right.$, and the anchoring energy $W \approx 10^{-5} \mathrm{Nm}^{-1}$ ), the Frank elasticity dominates the surface anchoring, which results in a more uniform NLC alignment without $a+1 / 2$ disclination. The periodic domain structure is highly associated with the presence of a disclination line; thus, the formation of a periodic domain structure is suppressed when $h$ or $\lambda$ are small.

Breaking symmetry of periodic domain structures. Next, we discuss several ways of breaking the symmetry between two neighbouring domains with opposite twist handedness and the consequences of introducing asymmetry. The twist handedness can be determined by introducing a chiral reagent, such as CB15, that induces righthanded twist (Fig. 2a-c). The liquid filament of the chiral nematic $\left(\mathrm{N}^{*}\right)$ with a right-handed twist distortion does not form a periodic domain structure (Fig. 2b, bottom). Immediately after the fusion of the $\mathrm{N}$ and $\mathrm{N}^{\star}$ liquid filaments, where the $\mathrm{CB} 15$ density gradient still exists, the fused liquid filaments show a continuous transition from the periodic domain structure to the right-handed twist region with no specific discontinuities (Fig. 2c). This demonstrates that $\mathrm{N}^{*}$ stabilizes one of the twisted domains in the periodic domain structure. The symmetry breaking also occurs when the angle between the easy axis of the NLC alignment and the groove direction deviates from a right angle, which is often observed at microwrinkle dislocations (Fig. 2d-e). In this case, one of the rotated disclination line segments is stabilized over the other because of the symmetry breaking, and thus, the formation of a periodic domain structure is suppressed. In both cases, one prescribed twist handedness dominates, indicating that the periodic domain structure can be controlled by changing the chiral strength of the NLC or the patterned alignment conditions.

Manipulation of colloids using zigzag defect lines. Disclination lines can trap small particles to reduce the total elastic energy of the system $^{26-28}$; the particles remove the energetically costly disclination cores. We indeed observed silica beads trapped at the disclination line at which the strongest elastic deformation is likely (Fig. 3). It should be noted that the particles cannot move freely along the disclination line, but are trapped selectively at its kinks. The kinks in the disclination line are presumably more energetically unfavourable 
a

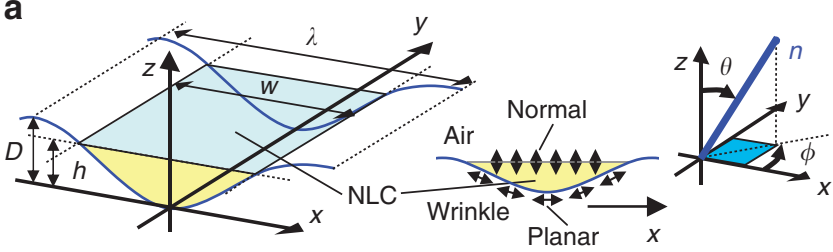

b

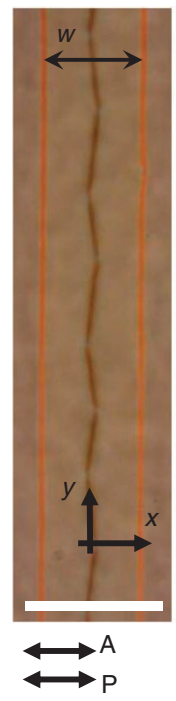

C

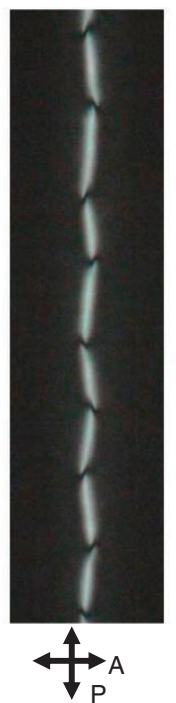

d

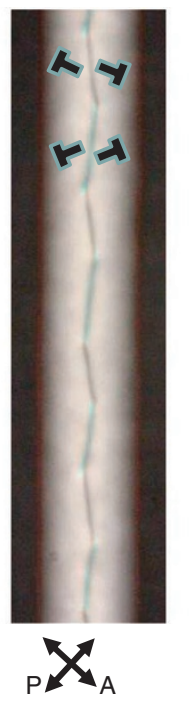

e

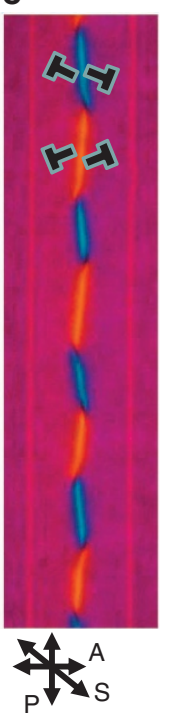

f

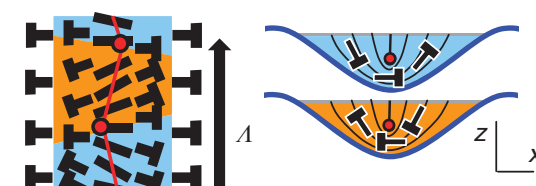

g

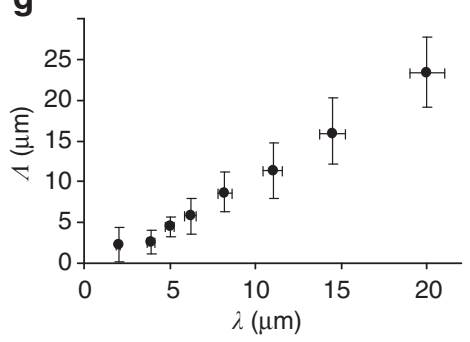

Figure 1 | Periodic alignment with a zigzag line defect in a NLC confined in microwrinkle grooves. (a) Geometrical confinement and hybrid anchoring conditions for a liquid filament of an NLC on a microwrinkle groove, where $D$ is the maximum depth of the groove, $h$ is the maximum thickness of the liquid filament, $\lambda$ is the wavelength of the microwrinkles, and $w$ is the width of the liquid filament. The blue curves indicate the profile of the sinusoidal groove. The definition of the polar and the azimuthal angles $\theta$ and $\phi$ of the local nematic director $\boldsymbol{n}$ (bold blue line) is also given. (b-e) Optical images with different configurations of the polariser (P) and analyser (A). Scale bar is $20 \mu \mathrm{m}$. In (e), a sensitive tint plate is used with the configuration shown as $\mathrm{S}$. The colour changes depending on the angle between the local $\boldsymbol{n}$ and the direction of $\mathrm{S}$; blue (orange) colour suggests that $\boldsymbol{n}$ is parallel (normal) to the direction of S. The nail symbols (T) indicate the projection of $\boldsymbol{n}$ onto the plane of the page (the head of the nail comes out of the page). (f) Schematic of the director structure $\boldsymbol{n}(\boldsymbol{r})$ with the definitions of the maximum twist angle $\phi$ and the rotation angle of the disclination line (red line) from the $y$ axis $\Omega$. $\Lambda$ is defined as the wavelength of the periodic domain structure. The red dots indicate disclination kinks. The blue (orange) colour indicate domains with $\phi<0(\phi>0)$, which corresponds to the colours in (e). The domain boundaries correspond to the black inclined line passing through the centre line of the groove in (c). (g) Plot of $\Lambda$ versus $\lambda$; mean \pm s.e.m. are shown and the number of samples for each point is over 50 . The data at $\lambda=2 \mu \mathrm{m}$ show larger error relative to the mean value, because the periodic domain structure is less regular. A periodic domain structure was not observed with $\lambda$ below $\sim 1.5 \mu \mathrm{m}$.

than the straight parts, which result in stronger binding of beads at the kinks. In addition, when the system is heated to the isotropic phase, the beads are no longer trapped and show random motion (Supplementary Movie 1). Our observation demonstrates that topological defects can have an active role in the control of colloidal assembly in LCs, whereas in usual LC colloids, topological defects are formed passively in response to colloid-surface anchoring ${ }^{19}$. Zigzag disclination lines can thus provide a novel way of manipulating colloidal particles.

\section{Discussion}

We discuss why a disclination line in a narrow groove can take a direction oblique to the groove, resulting in a zigzag shape. Following the discussion by Buscaglia et al. ${ }^{29}$ on the spontaneous twist deformations in an NLC under a straight disclination line, we assume that the dominant contributions to the total elastic energy of an NLC in the groove are from the elastic deformation in the cylindrical region around the disclination line, and from the twist deformation in the NLC below the cylindrical region. In a simplified case where $K_{11}=K_{33}=K$, the total elastic energy per unit groove length is given by:

$$
E_{\text {total }}(\Omega, \phi) \simeq \kappa \frac{\alpha_{c}+\sqrt{\cos ^{2}(\Omega+\phi)+\frac{K_{22}}{K} \sin ^{2}(\Omega+\phi)}}{\cos \Omega}+\frac{1}{2} \gamma \phi^{2}
$$

The first term is the contribution from the cylindrical region around the disclination line, where $\kappa \equiv(\pi / 4) K \ln \left(R / r_{c}\right), R$ is the radius of the cylinder, $r_{c}$ is the microscopic cut-off, and $\phi$ is the azimuthal angle of $\boldsymbol{n}$ at the bottom of the cylindrical region. The parameter $\alpha_{c}$, which is of the order of 0.1 , arises from the contribution inside the microscopic cut-off where the continuum Frank elasticity no longer holds. The second term accounts for the twist deformation below the cylindrical region. The symbol $\gamma$ is a monotonically increasing function of the anchoring strength at the groove surface, and takes a value between 0 and $K_{22} A / d^{2}$, where $A$ is the area of the region where the twist deformation is relevant, and $d$ is the distance between the bottom of the cylinder and the groove surface. Equation (1) indicates that a positive $\phi$ yields a smaller $E_{\text {total }}$ for $\Omega>0$ and vice versa. This is in agreement with our experimental findings described above. See Fig. 4a for the geometry, and Methods for theoretical details of the above and the following discussions. 
a

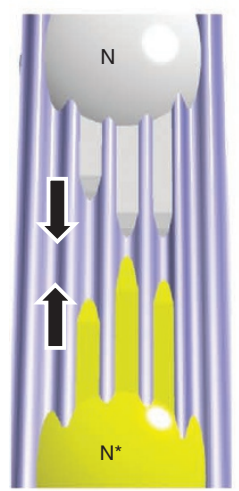

b

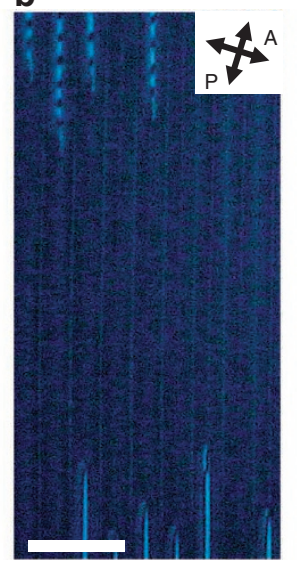

c

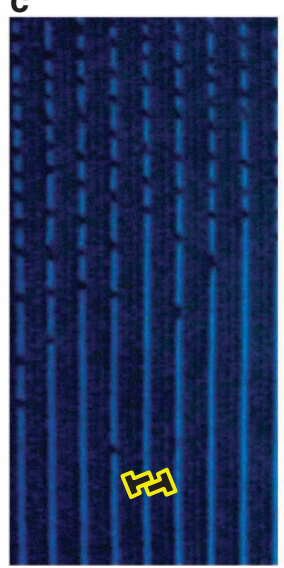

d

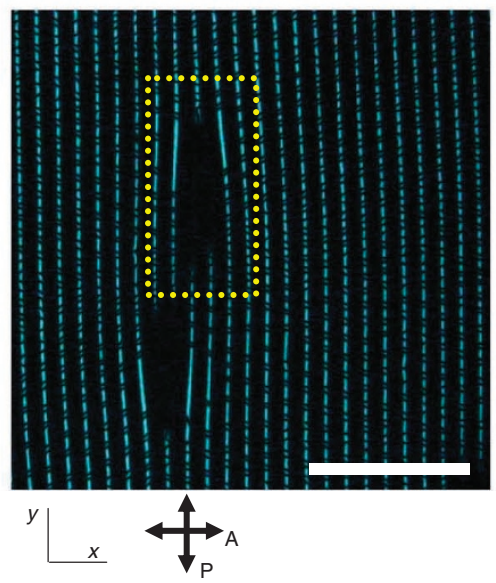

e

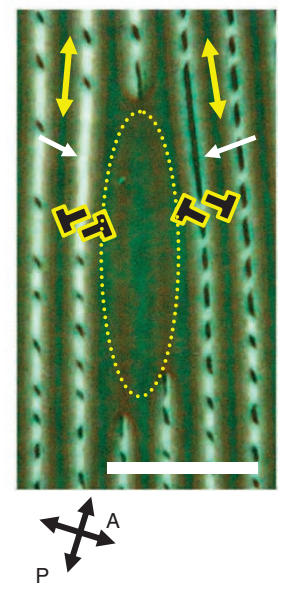

Figure $\mathbf{2}$ | Breaking the symmetry between two neighbouring domains with opposite twist handedness. (a) Schematic of the fusion process of liquid filaments protruding from the NLC and $N^{\star}$ LC droplets. The thick arrows indicate the direction of the capillary action. (b) Optical image of the liquid filaments before fusion. Note that the liquid filaments of $\mathrm{N}^{\star}$ do not form a periodic domain structure (bottom of (b)). Scale bar is $30 \mu \mathrm{m}$. (c) The fused liquid filaments of $\mathrm{N}$ and $\mathrm{N}^{\star}$ with the gradient of the chiral reagent. The nail symbols ( $\mathrm{T}$ ) indicate the projection of $\boldsymbol{n}$ onto the plane of the page (the head of the nail comes out of the page). (d) Optical image of the periodic domain structure in a large area that includes randomly produced dislocations of microwrinkle grooves. Scale bar is $50 \mu \mathrm{m}$. (e) Magnified image of the region indicated by the dotted rectangle in (d) with a slightly rotated polariser (P) and analyser $(A)$ to differentiate the domains with different twist handedness. The groove direction deviates slightly from the $y$ axis around the dislocation, as indicated by the yellow arrows. The absence of periodic domain structures is highlighted by the white arrows. The easy axis is in the horizontal direction. Liquid filaments are not formed at the dislocation, because the shallow grooves destabilize the liquid filament ${ }^{22}$ (dotted ellipse). Scale bar is $20 \mu \mathrm{m}$.

a

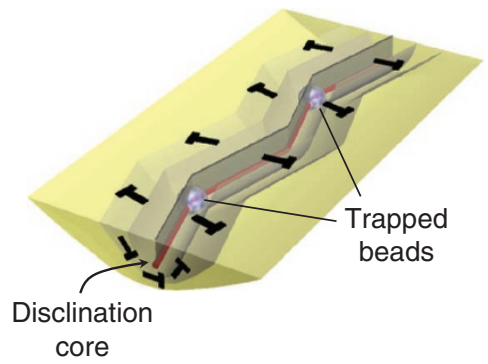

b

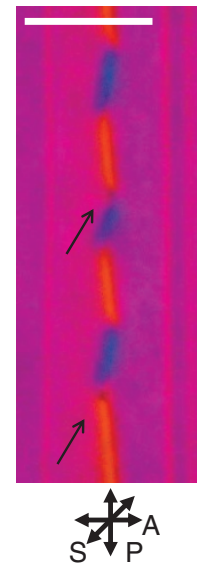

c

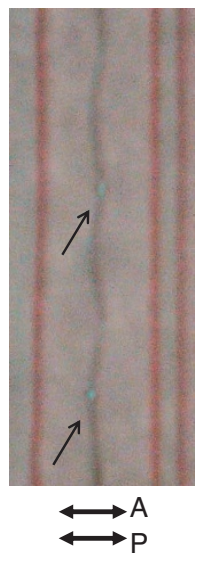

d

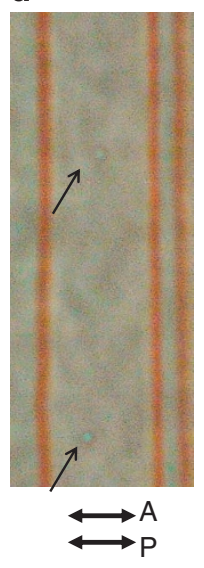

Figure 3 | Trapped silica beads at the domain boundaries of a periodic domain structure. (a) Schematic of the trapped beads at the kinks of the zigzag disclination line, or domain boundaries of the periodic domain structure. $(\mathbf{b}, \mathbf{c})$ Optical images of the periodic domain structure with two trapped silica beads at the disclination line kinks. The arrows indicate the positions of the beads. In (b), a tint plate (S) was used. The colour changes depending on the angle between the local $\boldsymbol{n}$ and the direction of S; blue (orange) colour suggests that $\boldsymbol{n}$ is parallel (normal) to the direction of S. (d) Optical image of the released silica beads just after the transition from a nematic to an isotropic phase (see also Supplementary Movie 1). The disclination line disappears and the beads are free to move in the isotropic liquid filament. The same part of the system is pictured in (b,c and $\mathbf{d})$. Scale bar is $15 \mu \mathrm{m}$.

A straight disclination line along the groove $(\Omega=0)$ becomes unstable when:

$$
\frac{\gamma}{\kappa}<\frac{\left(1+\alpha_{c}\right)\left(1-\frac{K_{22}}{K}\right)}{\alpha_{c}+\frac{K_{22}}{K}}
$$

In most of the NLCs of rod-like molecules, $K_{22}<K_{11}<K_{33}$ (ref. 30) and thus the right-hand side of equation (2) is positive. The numeri- cal analysis of equation (1) for various values of $K_{22} / K$ and $\gamma / \kappa$, given in Fig. $4 \mathrm{~b}-\mathrm{d}$, shows that $\Omega$ that gives the minimum of $E_{\text {total }}$ (referred to as $\Omega_{m}$ later) is largest at an intermediate value of $\gamma / \kappa$, or an intermediate anchoring strength at the groove surface, and that $\Omega_{m}$ can be larger for smaller values of $K_{22} / K$.

Note that a disclination line with $\Omega=0$ cannot be unstable when $K_{22}=K$ (so-called one-constant elasticity), or $\phi$ is rigidly fixed to zero (in both cases the first term of eq. (1) is a monotonically increasing function of $\Omega$ at $0 \leq \Omega<\pi / 2$ ). One can understand that this instabil- 
a
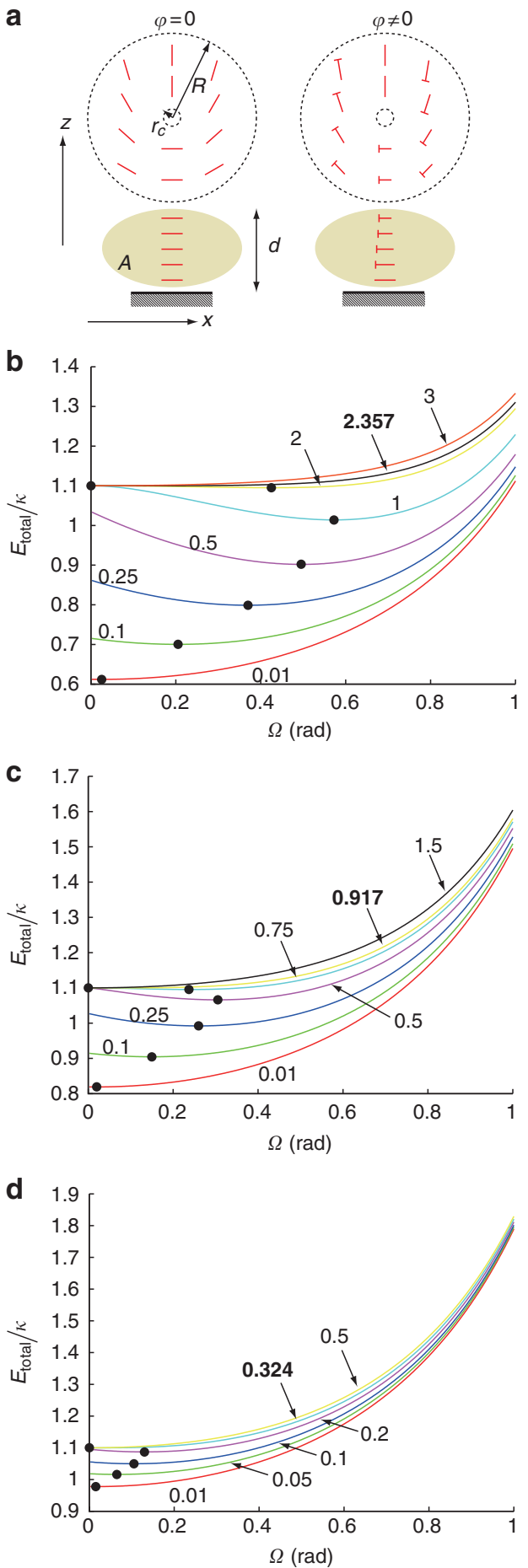

Figure 4 | Geometry of the system for the theoretical arguments and numerically evaluated free energy of the system. (a) Schematic of the geometry of the system. $R$ is the radius of the cylindrical region involving a disclination line. $r_{c}$ is the cut-off radius. The director profile $\boldsymbol{n}$ is given by nails in red (the head of the nail comes out of the page). When the twist angle $\varphi=0, \boldsymbol{n}$ lies in the $x-z$ plane, or the plane of the page (left), whereas non-zero $\varphi$ yields $\boldsymbol{n}$ out of the $x-z$ plane (right). In the region shaded in dark yellow, with area $A$ and height $d$, the twist distortion leading to equation (8) is present. The region shadowed by slashes is the bottom of the groove. (b-d) Rescaled energy of the system $E_{\text {total }}(\Omega) / \kappa$ as a function of $\Omega$ for $K_{22} / K=0.25$ (b), 0.5 (c), and 0.75 (d). Here, $\alpha_{c}$ in equation (1) is set to 0.1 . The numbers are the rescaled anchoring strength $\gamma / \kappa$. The points indicate the minimum of $E_{\text {total }}(\Omega) / \kappa$. The numbers in bold are the smallest $\gamma / \kappa$ violating equation (2). ity occurs because the elastic energy of the disclination line, the first term of equation (1), is reduced by adopting positive $\phi$, with the expense of the twist deformation below it (the second term of equation (1)). For this mechanism to be possible, the twist deformation cannot be energetically costly, requiring relatively small $K_{22}$ and thus small $\gamma / \kappa$. However, when $\gamma$ is so small (typically in the case of weak surface anchoring) that the second term of equation (1) is not effective, to minimize the first term, $\phi$ tends to be close to $\pi / 2-\Omega$. Then the denominator of the first term dominates the behaviour and again $\Omega_{m} \approx 0$, as seen from Fig. 4 ; the instability is effectively invisible. Thus, the instability of a disclination line along the groove direction resulting in finite $\Omega$ is a consequence of a delicate reduction of the disclination energy caused by finite $\phi$.

The disclination line takes alternate directions $\Omega_{m}$ and $-\Omega_{m}$ along the groove, both of which are allowed by symmetry, so that the disclination line can avoid strong elastic distortions of the director when it approaches the groove walls. This alternation results in the experimentally observed zigzag form. The wavelength $\Lambda$ of the zigzag can be roughly estimated by using the elastic energy associated with the approach of disclination line to the groove walls, and the elastic energy in narrow regions of width $l_{\mathrm{d}}$ from the abrupt change in direction of the disclination line. The former free energy, per unit length of the groove, is estimated as $f_{1} \simeq K(h / \lambda)\left(\Lambda \tan \Omega_{m} / \lambda\right)^{2}$, and the latter energy from the change in direction is roughly estimated as $f_{2} \simeq K l_{d} / \Lambda$. Numerical prefactors of order unity have been omitted. When $f_{1}+f_{2}$ is minimized with respect to $\Lambda, \Lambda \propto\left(\tan \Omega_{m}\right)^{-2 / 3}$ is obtained, and $\Lambda \propto \lambda\left(l_{\mathrm{d}} / h\right)^{1 / 3}$. The aspect ratio of the groove $\lambda / h$ is fixed experimentally to be almost constant ${ }^{22}$. If it is assumed that $l_{\mathrm{d}} \propto \lambda$, as expected in an NLC under strong surface anchoring where only the characteristic lengths of the geometrical confinement $(\lambda$ and $h$ ) determine the characteristic length scale of the elastic distortion $\left(l_{\mathrm{d}}\right)$, then $\Lambda \propto \lambda$, in agreement with experimental observations (Fig. 1g).

The self-organized topological structure of an NLC with a zigzag disclination line reported here is an interesting example of spontaneous symmetry-breaking phenomena arising from the coupling between material anisotropy, and asymmetric boundary conditions in a confined geometry. It may provide a new experimental system for studying basic and unexplored properties of topological defects not only in various LC phases, but also in other condensedmatter systems with a vectorial order. Our experiments show that our easy-to-make self-organized microwrinkles are quite useful for engineering the new zigzag disclination line structures and for manipulating small particles. The tunability of the shape of microwrinkles by simply exerting strain ${ }^{22,25}$ allows further dynamical control of the defect structure. Other efficient ways of controlling the defect structure would be the application of an electric or a magnetic field, and the tuning of LC elasticity by varying temperature or by changing the chirality and twisting power of the LC material. The variety of such technical advantages facilitates the micromanipulation of LC defect structures at a small scale with a very simple procedure. Our results demonstrate a new possibility of fine control of colloidal assembly without relying on conventional top-down lithographic techniques, and further knowledge of how the surface anchoring and the size of colloidal particles affect the trapping phenomena will sophisticate the manipulation of colloidal assembly using self-organized defects. Thus, our system with controllable geometry provides a route to a novel microfabrication method for colloidal assembly, optical patterning and guided polymerization and crystallization ${ }^{31}$, with its self-organized structure as a template.

\section{Methods}

Preparation of microwrinkles. Microwrinkles with aligned grooves were prepared as follows, which is identical to the method reported by Ohzono et al. ${ }^{22}$ A polydimethylsiloxane (PDMS) elastomer (Sylgard 184, Dow-corning) was cured 
on a smooth Si wafer. The smooth surface of the PDMS substrate $\left(12 \times 12 \times 6 \mathrm{~mm}^{3}\right)$ was weakly treated by an Ar plasma (SEDE-P, Meiwa Forsis) to enhance the wetting of $\mathrm{N}$-methylpyroridone (Sigma-Aldrich). Soon after an $\mathrm{N}$-methylpyroridone solution of poly-amic acid (Poly(pyromellitic dianhydride-co-4,4'-oxydianiline), Sigma-Aldrich) was coated on the activated PDMS surface using a spin-coater at 5000 r.p.m. for 90 s (MSA-150, Mikasa), the substrate was uniaxially compressed with the strain of approximately $-0.04(-4 \%)$. The sample was heated under compression at $180^{\circ} \mathrm{C}$ for $3 \mathrm{~h}$ to induce the transformation of poly(amic acid) to polyimide. During the temperature increase in this heating process, the PDMS sample dilated to the direction perpendicular to the compression due to thermal expansion. Thus, the main chains of the polymer plausibly aligned anisotropically, which imposed the observed alignment of the cyanobiphenyl NLCs. After cooling down to $25^{\circ} \mathrm{C}$ and the release of the compression, aligned microwrinkle grooves were formed with the easy axis of the LC alignment perpendicular to the groove direction. The wavelength of the groove $\lambda, 0.6 \sim 20 \mu \mathrm{m}$, was controlled by varying the concentration of the poly-amic acid solution from approximately 1 to $10 \mathrm{wt} \%$. The aspect ratio of the microwrinkles, $D / \lambda$, where $D$ is the groove depth, was almost constant, $0.13 \pm 0.01$.

Formation of liquid filaments of LCs. Liquid filaments were formed on the microgrooves, by placing a droplet of an NLC with a sub-millimetre diameter on the wrinkled surface. With the present aspect ratio, 5CB was spontaneously sucked into the microgrooves via capillary action ${ }^{22}$. Alternatively, the liquid filaments were formed by simply coating microwrinkles with 5CB (see Supplementary Fig. S1 for further details). The LC alignment was observed using a transmitted polarized microscope (BX-51P, Olympus). To differentiate small optical rotations visually by the colour difference, a 530-nm sensitive tint plate ( $1 \lambda$, U-TP530, Olympus) was used as a test plate compensator under crossed-Nicol conditions. $\mathrm{A} \mathrm{N}^{\star}$ phase doped with a right-handed chiral reagent, 4-cyano- $4^{\prime}-((S)$-2-methylbutyl)biphenyl (CB15 Merck, $\sim 3 \mathrm{wt} \%$ ) was used to investigate the effect of chirality. Droplets of $\mathrm{N}^{\star}$ and $\mathrm{N}$ (pure 5CB) LCs were placed a certain distance apart $(\sim 100 \mu \mathrm{m})$, and the liquid fila ments protruding from each droplet made contact on each microgroove and fused into liquid filaments connecting the droplets. A dispersion of melamine-coated silica beads with an average diameter of $\sim 600 \mathrm{~nm}$ (Optbeads, Nissan Chemical, $\sim 0.5 \mathrm{wt} \%)$ in $5 \mathrm{CB}$ was used for trapping colloids. The maximum thickness of the liquid filament, $h(>1.5 \mu \mathrm{m})$, was large enough for the silica beads to be fully immersed in 5CB without touching the bottom groove surface. We used a transmitted polarized optical microscope to observe the beads trapped at the domain boundaries.

Details of the theoretical arguments. For the derivation of equation (1), let us first explain the free energy of the disclination line evaluated in a cylindrical region of radius $R$. See Fig. 4 a for the geometry of the system. We consider a straigh disclination line uniform along the $y$ direction. When the disclination is a wedge disclination with the director $\boldsymbol{n}$ lying on a plane perpendicular to the disclination line (an $x-z$ plane), $\boldsymbol{n}=(\sin \theta, 0, \cos \theta)$, where $\theta$ is a function of $x$ and $z$. Now we are interested in the effect of the twist deformation on the energy of a disclination line, which we assume can be incorporated by a single variable $\varphi$ describing the rotation of $\boldsymbol{n}$ along the $z$ axis. We further assume that $\varphi$ is independent of $x, y$ and $z$. Then, the director field can be given by $n=(\sin \theta \cos \varphi, \sin \theta \sin \varphi$, $\cos \theta)$. The Frank free-energy density $f_{\text {Frank}}$, under the simplifying assumption of $K_{11}=K_{33}=K$, is

$$
f_{\mathrm{Frank}}=\frac{1}{2} K\left[\left(\frac{\partial \theta}{\partial \tilde{x}}\right)^{2}+\left(\frac{\partial \theta}{\partial z}\right)^{2}\right]
$$

with $\tilde{x} \equiv x / \sqrt{\cos ^{2} \varphi+\left(K_{22} / K\right) \sin ^{2} \varphi}$. A familiar solution involving a disclination line of strength $+1 / 2$ that minimizes the Frank elastic energy $\int d r f_{\text {Frank }}$ is $\theta=\theta_{0}+(1 / 2) \arctan (z / \tilde{x})$ with $\theta_{0}$ being an integration constant, and thus $f_{\text {Frank }}=(K / 8)\left(\tilde{x}^{2}+z^{2}\right)^{-1}$. The free energy of a disclination line per unit length is then expressed as

$$
\begin{aligned}
E_{\text {disclination }} & =E_{c}+\frac{K}{8} \int_{r_{c}}^{R} d r \int_{0}^{2 \pi} d \chi \frac{2}{\left(\frac{\cos \chi}{\sqrt{\cos ^{2} \varphi+\left(K_{22} / K\right) \sin ^{2} \varphi}}\right)^{2}+\sin ^{2} \chi} \\
& =E_{c}+\left(\frac{\pi K}{4} \ln \frac{R}{r_{c}}\right) \sqrt{\cos ^{2} \varphi+\left(K_{22} / K\right) \sin ^{2} \varphi}
\end{aligned}
$$

where we have introduced a transformation of variables $x=r \cos \chi$ and $z=r \sin \chi$, together with a microscopic cut-off $r_{c}$. The contribution $E_{\mathcal{C}}$, of the order of $K$ (ref. 1), comes from the region $r<r_{c}$ in which the description of the orientational order in terms of $\boldsymbol{n}$ is no longer valid. In the case of our interest, $\ln \left(R / r_{c}\right) \simeq 10$ (ref. 1), and thus $E_{c}=\alpha_{c} \kappa$ with $\alpha_{c} \simeq 0.1$. When the disclination is not oriented along the $y$ axis (groove direction) and the angle between the disclination and the $y$ axis is $\Omega$ (Fig. 1f), the free energy of the disclination line per unit length along the groove direction is $E_{\text {disclination }} / \cos \Omega$. Moreover, when the azimuthal angle of $\boldsymbol{n}$, the angle between $\boldsymbol{n}$ and the $x$ axis, is $\phi$ at the bottom, $\varphi=\Omega+\phi$. Thus,

$$
\frac{E_{\text {disclination }}}{\cos \Omega}=\kappa \frac{\alpha_{c}+\sqrt{\cos ^{2}(\Omega+\phi)+\frac{K_{22}}{K} \sin ^{2}(\Omega+\phi)}}{\cos \Omega},
$$

with $\kappa \equiv(\pi K / 4) \ln \left(R / r_{c}\right)$, yielding the first term of $E_{\text {total }}$, equation (1). We note here that the form of the disclination energy presented in ref. 29 is different from ours, equation (4). This is because Buscaglia et al. ${ }^{29}$ made an oversimplifying assumption for the profile of $\boldsymbol{n}$ around a disclination; in our notation, they employed $\theta=(1 / 2) \arctan (z / x)$, valid only when $K_{11}=K_{22}=K_{33}$, and applied the profile to the general cases of $K_{11} \neq K_{22} \neq K_{33}$.

In the evaluation of the elastic energy due to the twist distortion below the cylindrical region containing the disclination line, we make a crude assumption that the twist distortion there is present in an area of $A$ at a cross section parallel to the $x-z$ plane, and that $l$ is the width of the groove bottom in contact with the NLC. The azimuthal angle of $\boldsymbol{n}$ at the groove bottom is referred to as $\phi_{b}$. Then the free energy associated with the twist distortion and that with surface anchoring at the groove bottom are, per unit length along the groove direction,

$$
\begin{aligned}
E_{\text {twist }} & \simeq \frac{1}{2} K_{22} A\left(\frac{\phi-\phi_{b}}{d}\right)^{2}, \\
E_{\text {anchoring }} & \simeq \frac{1}{2} W l \sin ^{2} \phi_{b} \simeq \frac{1}{2} W l \phi_{b}^{2} .
\end{aligned}
$$

In equation (6), $d$ is the distance between the bottom of the cylindrical region containing the disclination and the groove bottom, throughout which the twist distortion is present. In equation (7), W implies the anchoring strength, and it is assumed that $\left|\phi_{b}\right| \ll 1$. By substituting the solution of $\partial\left(E_{\text {twist }}+E_{\text {anchoring }}\right) / \partial \phi_{b}=0$ as an equation for $\phi_{b}$ into equations (6) and (7), one can eliminate $\phi_{b}$, and obtain

$$
E_{\text {twist }}+E_{\text {anchoring }} \simeq \frac{1}{2} \gamma \phi^{2}
$$

constituting the second term of $E_{\text {total }}$, equation (1), with $\gamma \equiv\left(K_{22} W l A / d^{2}\right) /\left(K_{22} A /\right.$ $\left.d^{2}+W l\right)$. One can easily see that $\gamma$ is a monotonically increasing function from 0 to $K_{22} A / d^{2}$ with increasing $W$ from zero to infinity.

For the derivation of equation (2), we expand equation (1) up to second order in $\Omega$ in the numerator of the first term, and in $\Omega+\phi$ in its denominator. The result is

$$
E_{\text {total }}^{(2)}=\kappa\left[\frac{1}{2}\left(1+\alpha_{c}\right) \Omega^{2}+\frac{1}{2}\left(\frac{K_{22}}{K}-1\right)(\Omega+\phi)^{2}\right]+\frac{1}{2} \gamma \phi^{2} .
$$

Here the superscript (2) is to emphasize that the expansion is kept up to the second order. By eliminating $\phi$ after solving $\partial E_{\text {total }}^{(2)} / \partial \phi=0$ as an equation for $\phi$, and substituting the solution to equation (9), one obtains

$$
E_{\text {total }}^{(2)}=\frac{1}{2} \kappa\left(1+\alpha_{c}-\left(1-\frac{K_{22}}{K}\right) \frac{\gamma}{\gamma-\kappa\left(1-\frac{K_{22}}{K}\right)}\right) \Omega^{2} .
$$

Thus, it can be shown that $\Omega=0$ becomes unstable when equation (2) is fulfilled.

To find $\Omega$ that minimizes $E_{\text {total }}$ (referred to as $\Omega_{m}$ later), numerical calculations are necessary. In Fig. 4b-d, we plot $E_{\text {total }}(\Omega)$ for several values of $\gamma / \kappa$ and $K_{22} / K$ after the elimination of $\phi$ by solving $\partial E_{\text {total }} / \partial \phi=0$ and substituting the solution to equation (1), the same procedure as in the above discussion. One can find that the range of $\gamma / \kappa$ in which $\Omega_{m}$ is non-zero becomes smaller with the increase of $K_{22} / K$ towards 1 (as also seen from eq. (2)). It is also clearly seen that when $\gamma / \kappa$ approaches zero, corresponding to the limit of weak anchoring, $\Omega_{m}$ approaches zero as well, and that the largest $\Omega_{m}$ for fixed $K_{22} / K$, which becomes larger with the decrease of $K_{22} / K$, is attained at some intermediate value of $\gamma / \kappa$, or intermediate surface anchoring at the groove bottom.

Finally, we estimate $f_{1}$ necessary for the evaluation of $\Lambda$. The elastic energy associated with the approach of the disclination line to the groove walls, per unit length of the groove, is estimated as

$$
f_{1}(y) \simeq \frac{1}{2} c_{1} K h\left\{\frac{1}{\lambda / 2-u(y)}+\frac{1}{\lambda / 2+u(y)}\right\}
$$

where $u(y)$ is the displacement of the disclination line from the centre of the groove, and $c_{1}$ is a numerical constant of order unity. Equation (11) is composed of the elastic energies in a region between the disclination line and one of the walls, involving the distortion of the director $\boldsymbol{n}$ with a characteristic length $\lambda / 2-u(y)$, and in another region between the disclination line and the other wall 
whose distance is $\lambda / 2+u(y)$. The assumption that the disclination line is made up of a straight line segment $u(y)=y \tan \Omega_{m}$ at $-\Lambda / 4<y<\Lambda / 4$ yields the spatial average of $f_{1}$ as

$$
\begin{aligned}
\bar{f}_{1} & \equiv \frac{1}{\Lambda / 2} \int_{-\Lambda / 4}^{\Lambda / 4} d y f_{1} \\
& =\frac{2 c_{1} K h}{\Lambda \tan \Omega_{m}} \ln \frac{2 \lambda+\Lambda \tan \Omega_{m}}{2 \lambda-\Lambda \tan \Omega_{m}} \\
& \simeq \frac{2 c_{1} K h}{3 \lambda}\left(\frac{\Lambda \tan \Omega_{m}}{2 \lambda}\right)^{2},
\end{aligned}
$$

resulting in the form of $f_{1}$ given in Discussion apart from a numerical factor (in Discussion, the overbar of $\bar{f}_{1}$ has been omitted).

\section{References}

1. de Gennes, P. G. \& Prost, J. Physics of Liquid Crystals (Oxford University Press, 1993).

2. Lavrentovich, O. D. \& Pergamenshchik, V. M. Patterns in thin liquid crystal films and the divergence ('surfacelike') elasticity. Int. J. Mod. Phys. B 9, 2389-2437 (1995).

3. Manyuhina, O. V., Cazabat, A. M. \& Ben Amar, M. Instability patterns in ultrathin nematic films: comparison between theory and experiment. Europhys. Lett. 92, 16005 (2010).

4. Link, D. R., Nakata, M., Takanishi, Y., Ishikawa, K. \& Takezoe, H. Patterns in hybrid nematic liquid-crystal films: topography and topology. Phys. Rev. Lett. 87, 195507 (2001).

5. Palffy-Muhoray, P., Gartland, E. C. \& Kelly, J. R. A new configurational transition in inhomogeneous nematics. Liq. Cryst. 16, 713-718 (1994).

6. Lavrentovich, O. D. Geometrical anchoring at an inclined surface of a liquid crystal. Phys. Rev. A 46, R722-R725 (1994).

7. Carbone, G. et al. Mechanically induced biaxial transition in a nanoconfined nematic liquid crystal with a topological defect. Phys. Rev. Lett. 103, 167801 (2009).

8. Meyer, R. B. On the existence of even indexed disclinations in nematic liquid crystals. Phil. Mag. 27, 405-424 (1973).

9. Mihailovic, M. \& Oswald, P. Zigzag disclination in uniaxial nematic phases: study in capillary tubes. P. J. Phys. France 49, 1467-1475 (1988).

10. Oswald, P. \& Pieranski, P. Nematic and Cholesteric Liquid Crystals (Taylor \& Francis, New York, 2005).

11. Lavrentovich, O. D. Topological defects in dispersed words and worlds around liquid crystals, or liquid crystal drops. Liq. Cryst. 16, 117-124 (1998).

12. Yamamoto, J. \& Tanaka, H. Transparent nematic phase in a liquid-crystal-based microemulsion. Nature 409, 321-325 (2001).

13. Fukuda, J. \& Žumer, S. Quasi-two-dimensional Skyrmion lattices in a chiral nematic liquid crystal. Nat. Commun. 2, 246 (2011)

14. Fernández-Nieves, A. et al. Novel defect structures in nematic liquid crystal shells. Phys. Rev. Lett. 99, 157801 (2007).

15. Lee, B.- W. \& Clark, N. A. Alignment of liquid crystals with patterned isotropic surfaces. Science 291, 2576-2580 (2001).

16. Kim, J.- H., Yoneya, M. \& Yokoyama, H. Tristable nematic liquid-crystal device using micropatterned surface alignment. Nature 420, 159-162 (2002).

17. Ferjani, S., Choi, Y., Pendery, J., Petschek, R. G. \& Rosenblatt, C. Mechanically generated surface chirality at the nanoscale. Phys. Rev. Lett. 104, 257801 (2010).

18. Nelson, D. R. Toward a tetravalent chemistry of colloids. Nano Lett. 2, 1125-1129 (2002)

19. Tkalec, U., Ravnik, M., Čopar, S., Žumer, S. \& Muševič, I. Reconfigurable knots and links in chiral nematic colloids. Science 333, 62-65 (2011).
20. Mermin, N. D. The topological theory of defects in ordered media. Rev. Mod. Phys. 51, 591-648 (1979).

21. Kleman, M. \& Lavrentovich, O. D. Soft Matter Physics:An Introduction (Springer-Verlag, New York, 2003).

22. Ohzono, T., Monobe, H., Shiokawa, K., Fujiwara, M. \& Shimizu, Y. Shaping liquid on a micrometre scale using microwrinkles as deformable open channel capillaries. Soft Matter 5, 4658-4664 (2009).

23. Bowden, N. et al. Spontaneous formation of ordered structures in thin films of metals supported on an elastomeric polymer. Nature 393, 146-149 (1998).

24. Genzer, J. \& Groenewold, J. Soft matter with hard skin: from skin wrinkles to templating and material characterization. Soft Matter 2, 310-323 (2006).

25. Ohzono, T., Monobe, H., Yamaguchi, R., Shimizu, Y. \& Yokoyama, $H$. Dynamics of surface memory effect in liquid crystal alignment on reconfigurable microwrinkles. Appl. Phys. Lett. 95, 014101 (2009).

26. Voloschenko, D., Pishnyak, O. P., Shiyanovskii, S. V. \& Lavrentovich, O. D. Effect of director distortions on morphologies of phase separation in liquid crystals. Phys. Rev. E 65, 060701(R) (2002).

27. Ravnik, M., Alexander, G. P., Yeomans, J. M. \& Žumer, S. Three-dimensional colloidal crystals in liquid crystalline blue phases. Proc. Natl Acad. Sci. USA 108, 5188-5192 (2011).

28. Yoon, D. K. et al. Internal structure visualization and lithographic use of periodic toroidal holes in liquid crystals. Nature Mater. 6, 866-870 (2007).

29. Buscaglia, M., Lombardo, G., Cavalli, L., Barberi, R. \& Bellini, T. Elastic anisotropy at a glance: the optical signature of disclination lines. Soft Matter 6, 5434-5442 (2010).

30. Madhusudana, N. V. \& Pratibha, R. Elasticity and orientational order in some cyanobiphenyls: part IV. Reanalysis of the data. Mol. Cryst. Liq. Cryst. 89, 249-257 (1982).

31. Nishimura, T., Ito, T., Yamamoto, Y., Yoshio, M. \& Kato, T. Macroscopically ordered polymer $/ \mathrm{CaCO}_{3}$ hybrids prepared by using a liquid-crystalline template. Angew. Chem. Int. Ed. 47, 2800-28003 (2008).

\section{Acknowledgements}

We thank M. Yoneya, J. Yamamoto and Y. Kimura for useful discussions, and T. Yamamoto for supplying the chiral reagent. This work was carried out under the auspices of the New Energy and Industrial Technology Development Organization (NEDO) of Japan under the Industrial Technology Research Grant Program in 2008. We were supported also by Grants-in-Aid (KAKENHI) for Young Scientists (B).

\section{Author contributions}

T. O. conceived the central idea, performed the experiments and analysed the results. J. F. contributed to the theoretical argument. Both discussed the results and wrote the manuscript.

\section{Additional information}

Supplementary Information accompanies this paper at http://www.nature.com/ naturecommunications

Competing financial interests: The authors declare no competing financial interests.

Reprints and permission information is available online at http://npg.nature.com/ reprintsandpermissions/

How to cite this article: Ohzono, T., \& Fukuda, J. Zigzag line defects and manipulation of colloids in a nematic liquid crystal in microwrinkle grooves. Nat. Commun. 3:701 doi: 10.1038/ncomms1709 (2012).

License: This work is licensed under a Creative Commons Attribution-NonCommercialNoDerivative Works 3.0 Unported License. To view a copy of this license, visit http:// creativecommons.org/licenses/by-nc-nd/3.0/ 